\title{
Ionization in a Bose-Einstein condensate of metastable Helium: a quantitative monitoring tool
}

\author{
A. Aspect, O. Sirjean, S. Seidelin, J. Viana Gomes, D. Boiron and C. I. Westbrook \\ Laboratoire Charles Fabry de l'Institut d'Optique, UMR 8501 du CNRS, B.P. 147, \\ F-91403 ORSAY CEDEX, FRANCE
}

\begin{abstract}
Residual ionization in a BEC of $2{ }^{3} \mathrm{~S}_{1}$ metastable Helium $\left(\mathrm{He}^{*}\right)$, can be used to monitor in real time the evolution of a single sample of condensate. We use this method, combined to Time Of Flight measurements, to study the decay of the condensate. It has also allowed us to measure 2-body and 3-body ionizing collisions rate constants. This method should allow for the study of the growth of a condensate, in particular in the hydrodynamic regime. We point out the importance of quantum depletion in $\mathrm{He}^{*}$ BEC's.
\end{abstract}

\section{Introduction}

The achievement of Bose-Einstein condensation of metastable helium (He in the $2{ }^{3} \mathrm{~S}_{1}$ state denoted $\left.\mathrm{He}^{*}\right)[1,2]$ constituted a pleasant surprise for experimentalists and a remarkable vindication for the theorists who predicted the possibility [3]. Success hinged, among other things, on a strong suppression of Penning ionization in the (spinpolarized) magnetic trap. The ionization rate is not completely suppressed however, and when the atomic density gets high enough, a cold, magnetically trapped sample of $\mathrm{He}^{*}$ does produce a detectable flux of ions. As shown in [1], this signal can be used to monitor in real time the appearance and the decay of a single condensate. This opens the exciting prospect to study the growth kinetics of a condensate [4], provided that we can quantitatively interpret the ion rate. This requires a precise knowledge of the ionization rate constants.

In this presentation, we show how we can combine this ion monitoring with time of flight (TOF) measurements to extract quantitative data. We have first studied the rapid decay of a condensate after its formation, and shown that, by application of a $\mathrm{RF}$ knife, we can keep a quasi pure condensate for as long as 10 seconds. We have then been able to study the residual ionization in such quasi pure BEC's, and to extract 2 and 3 body rate constants without relying on fits to non-exponential decay of the number of atoms, which are notoriously difficult to interpret quantitatively [5] and which require a good stability of the experiment $[5,6,7]$. Finally, a comparison of the decay associated with ionization to the global decay curve allows for a consistency test of our results, and it reveals no evidence for collisional avalanche processes, in contrast with the case of ${ }^{87} \mathrm{Rb}[8]$.

We show in conclusion how this work suggests that $\mathrm{He}^{*}$ seems to be a good candidate for studying "hydrodynamic" regime as well as the effects of quantum depletion $[9]$. 


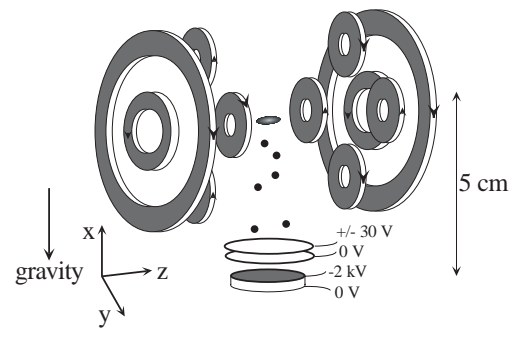

Figure 1: Experimental setup. The cold atoms are trapped in a cloverleaf type magnetic trap. A special feature of our set up is the microchannel plate detector $(M C P)$ placed below the trapping region. Two grids above the MCP allow us either to repel positive ions and detect only the He* atoms suddenly released from the trap (Time Of Flight measurements), or to attract and detect the positive ions produced in the trapped cloud (ion rate measurements).

\section{Apparatus and experimental procedure}

Much of our setup has been described previously $[1,10,11,12]$. Briefly, we trap up to $2 \times 10^{8}$ atoms at $1 \mathrm{mK}$ in a Ioffe-Pritchard trap with a lifetime $(\tau)$ of $90 \mathrm{~s}$. We use a "cloverleaf" configuration (Fig.1) [13] with a bias field $B_{0}=150 \mathrm{mG}$. The axial and radial oscillation frequencies in the harmonic trapping potential are typically $\nu_{\|}=47 \pm 3 \mathrm{~Hz}$ and $\nu_{\perp}=1800 \pm 50 \mathrm{~Hz}$ respectively $\left(\bar{\omega} / 2 \pi=\left(\nu_{\|} \nu_{\perp}^{2}\right)^{1 / 3}=534 \mathrm{~Hz}\right) . \mathrm{A}$ special feature of our set up is the detection scheme, based on a 2 stage, single anode microchannel plate detector (MCP) placed below the trapping region (Fig.1). Two grids above the MCP allow us either to repel positive ions and detect only the He* atoms, or to attract and detect positive ions produced in the trapped cloud.

To detect the ion flux, the MCP is used in counting mode: the anode pulses from each ion are amplified, discriminated with a $600 \mathrm{~ns}$ dead time and processed by a counter which records the time delay between successive events. Typical count rates are between $10^{2}$ and several $10^{4} \mathrm{~s}^{-1}$. At such rates, we expect no saturation effect provided that the ions impacts are spread over the whole MCP. We have checked that the correlation function of the count rate is flat, indicating that there is no double counting nor any significant positive or negative time correlation in the ion production. The dark count rate is of order $1 \mathrm{~s}^{-1}$. By changing the sign of the grid voltage, we have checked that while counting ions, the neutral $\mathrm{He}^{*}$ detection rate is negligible compared to the ion rate (less than $5 \%$ ) even when the radio frequency ( $\mathrm{rf}$ ) shield is on. We estimate the ion detection efficiency by assuming that only ions which hit the open channels of the MCP (60\% of the total area) are detected (with a $100 \%$ quantum efficiency). We then multiply by the transmission of the two grids $(0.84)^{2}$. Based on Refs. $[14,15]$, we assume this $(0.42)$ is an upper limit of our detection efficiency.

To find the values of the atom number $N_{0}$ and peak density $n_{0}$ of the BEC corresponding to a particular measured ion rate, as well as the number of atoms and temperature in the thermal cloud, we use the MCP to observe the time-of-flight signal 


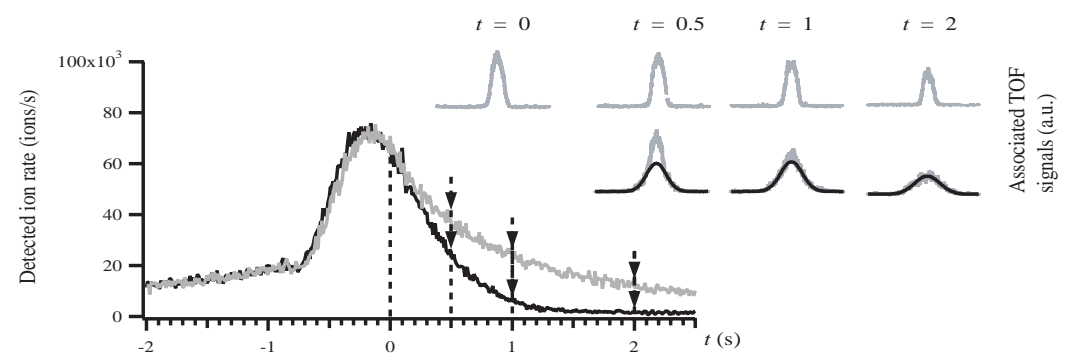

Figure 2: Birth and decay of a $H e^{*} B E C$, monitored by detection of the ion current from the trapped atoms. The evaporative cooling rf-knife frequency is ramped down until $t=0$, and the rf-knife is either turned off (dark plot), or kept on at the end-of-the-ramp frequency (grey plot). Each of these two plots correspond to a single realization of a condensate, and can be repeated in a reproducible way (the reproducibility is easily checked on the first part of the plot). Examples of TOF at various delays, corresponding to repeated runs in either situation, are displayed. They reveal that in the absence of rf-knife the sample rapidly heats up, and the condensed fraction decreases while the thermal cloud grows(lower series of TOF's: the dark curve represents the thermal cloud, fitted to the wings). In contrast, when the rf-kife is kept on, the condensate remains quasi pure (upper series of TOF's).

(TOF) of the He* atoms released from the rapidly switched off trap. The instantaneous count rate can be as high as $10^{6} \mathrm{~s}^{-1}$, and the MCP saturates when used in counting mode. To avoid this problem, we lower the MCP gain, and record the TOF signal in analog mode with a time constant of 400 microseconds. Several tests have shown that the detector behavior is then linear. We fit the TOF signals to the sum of a Bose function (integrated over transverse dimensions) describing the thermal cloud, and the inverted parabola squared expected for a BEC in the Thomas-Fermi regime [1]. We have checked that the chemical potential $\mu$ varies as $N_{0}^{2 / 5}$ as expected, over almost 2 decades in atom number. Residuals from the linear fit do not show any systematic variation. A fit on a log-log plot gives a slope of 0.39 . These observations are a good indication of the detection linearity.

\section{Decay of a $\mathrm{He}^{*}$ condensate}

In a typical run, forced evaporative cooling takes place for $40 \mathrm{~s}$, down to an rf-knife frequency of $500 \mathrm{KHz}$, about $50 \mathrm{kHz}$ above the minimum of the trapping potential. Near the end of the ramp, the ion rate increases sharply, signaling the appearance of a BEC (Fig.2). After reaching the final value, we either turn off the rf-knife (dark plot in Fig.2), or hold it on at the final frequency of the evaporative ramp (grey plot in Fig.2). In either case, we then monitor the ion rate until the time when we choose to switch off the trap and observe the TOF.

The plots of the ion rate in Fig.2 first reveal that the condensate survives much 
longer with the rf-knife kept on, which was to be expected. In addition, the TOF's realized at various stages of the decay, clearly show that the rapid decay of the condensate in the absence of the rf-knife is dominated by a heating: a fit to the wings of the TOF shows that the thermal cloud rapidly grows, and the temperature increases by about $300 \mathrm{nK} / \mathrm{s}$, while the condensed fraction simultaneously decreases, so that the total number of atoms is approximatively constant. This heating is suppressed when the rf-knife is kept on (rf-shield), as already observed with alkalis.

The understanding of the rise and decay of the ion rate, in a situation where the total number of trapped atoms does not vary rapidly, relies on the assumption that the ion rate increases with the density for a constant total number of atoms. This means that the ion production is not dominated by collisions with the background gas, but rather by collisions between trapped $\mathrm{He}^{*}$ atoms, that may be 2-body or 3-body ionizing collisions.

\section{Two and three-body ionization rate constants}

Much theoretical $[3,16]$ and experimental $[17,18,1,2]$ work has already been devoted to estimating inelastic decay rates in He*. The dominant 2-body decay mechanisms, which we will refer to collectively as Penning ionization,

$$
H e^{*}+H e^{*} \rightarrow\left\{\begin{array}{l}
H e^{+}+H e(1 S)+e^{-} \\
H e_{2}^{+}+e^{-}
\end{array}\right.
$$

are known to be suppressed by at least 3 orders of magnitude in a spin-polarized sample, but the total rate constant has not yet been measured. The 3-body reaction,

$$
H e^{*}+H e^{*}+H e^{*} \rightarrow \quad \begin{array}{ll}
H e_{2}^{*} & +H e^{*}(\sim 1 m K) \\
\hookrightarrow & H e^{+}+H e(1 S)+e^{-}
\end{array}
$$

proceeds via 3-body recombination followed by autoionization of the excited molecule. All reactions yield one positive ion which can easily be detected in our apparatus. We define the 2-body and 3-body ionizing collision rate constants $\beta$ and $L$ according to the density loss in a thermal gas [21]: $\frac{d n}{d t}=-\frac{n}{\tau}-\beta n^{2}-L n^{3}$ with $n$ the local density; $\tau$ is the (vacuum limited) lifetime of the sample. The theoretical estimate of the rate constants at $1 \mu \mathrm{K}$ are $\beta \sim 2 \times 10^{-14} \mathrm{~cm}^{3} \mathrm{~s}^{-1}[3,16]$ and $L \sim 10^{-26} \mathrm{~cm}^{6} \mathrm{~s}^{-1}$ [19].

For a pure condensate in the Thomas-Fermi regime with a number of atoms $N_{0}$, and a peak density $n_{0}$, we can then derive an expected ionization rate per trapped atom $(\Gamma)$ :

$$
\Gamma=\frac{\text { Ion rate }}{N_{0}}=\frac{1}{\tau^{\prime}}+\frac{2}{7} \kappa_{2} \beta n_{0}+\frac{8}{63} \kappa_{3} L n_{0}^{2} .
$$

The numerical factors come from the integration over the parabolic spatial profile and the fact that although 2 or 3 atoms are lost in each type of collision, only 1 ion is produced. The effective lifetime $\tau^{\prime} \geq \tau$ is due to ionizing collisions with the background gas. The factors $\kappa_{2}$ and $\kappa_{3}$ take into account the fact that the 2 and 3 -particle local correlation functions are smaller than those of a thermal cloud. For a dilute $\mathrm{BEC}$, one has $\kappa_{2}=1 / 2$ ! and $\kappa_{3}=1 / 3$ ! [9, 7]. Because the He* scattering length is so large, quantum depletion can lead to significant corrections [9] to the $\kappa$ 's as we discuss below.

To determine the ionizing collision rate constants $\beta$ and $L$, we need an absolute calibration of the number of atoms and the peak density. As discussed in Ref. [1], all the 


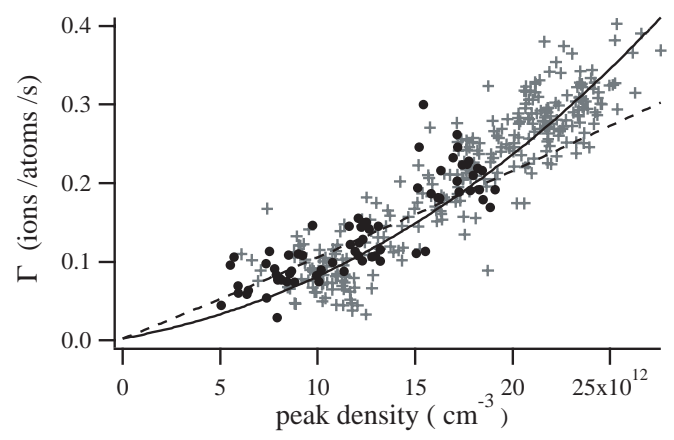

Figure 3: Ion rate per trapped atom versus peak density for different quasi-pure $B E C$ 's. The atom number is varied by waiting for different delays, with the rfshield on. The plot involves different set of data, taken for 2 different trapping configurations, corresponding to different average frequencies: $\nu_{\perp}=1800 \mathrm{~Hz}$ (crosses) and $\nu_{\perp}=1200 \mathrm{~Hz}$ (circles). The dashed line corresponds to the best fit involving only 2-body collisions. The solid line is a fit to 2 and 3-body processes.

atoms are not detected, and the direct calibration has a $50 \%$ uncertainty which is responsible for the large uncertainty in the scattering length $a$. In fact the measurement of the chemical potential gives an accurate value for the product $n_{0} a=\mu m / 4 \pi \hbar^{2}$, and with the value of $\bar{\omega}$ gives the product $N_{0} a=(1 / 15)(\hbar / m \bar{\omega})^{1 / 2}(2 \mu / \hbar \bar{\omega})^{5 / 2}$ as well. We use this method to measure $n_{0}$ and $N_{0}$, taking $a=20 \mathrm{~nm}$. With the present uncertainty on $a(8 \mathrm{~nm} \leq a \leq 30 \mathrm{~nm}[1,2])$ this entails a large uncertainty on $n_{0}$ and $N_{0}$, but it is likely that the $\mathrm{He}^{*}$ scattering length will be measured more accurately in the future. It will be then easy to reprocess our data to obtain more precise values of the ionization rate constants.

Figure 3 shows the ion rate per atom $\Gamma$ versus the peak density $n_{0}$. The densest sample corresponds to $N_{0}=2 \times 10^{5}$ atoms and $n_{0}=2.5 \times 10^{13} \mathrm{~cm}^{-3}$. The corresponding Thomas-Fermi radii are $r_{\perp} \simeq 5 \mu \mathrm{m}$ and $r_{\|} \simeq 200 \mu \mathrm{m}$. The vertical intercept in Fig. 3 corresponds to ionizing collisions with the background gas $\left(1 / \tau^{\prime}\right)$. It is negligible at the scale of the figure.

The curvature seen in Fig. 3 shows that 3 -body ionizing collisions are not negligible. A fit to equation 3 yields values of $\beta$ and $L$ of the order of $3 \times 10^{-14} \mathrm{~cm}^{3} \mathrm{sec}^{-1}$ and $10^{-26} \mathrm{~cm}^{6} \mathrm{sec}^{-1}$, respectively, with relative uncertainties of about $60 \%$. These values are in good agreement with the theoretical estimates.

To get final values of $\beta$ and $L$, we must correct the raw fitted values for several effects. First, for 3-body collisions, quantum depletion is important. For $T=0$, reference [9] gives a multiplicative correction to the factor $\kappa_{3}$ of the form $(1+\epsilon)$ where $\epsilon$ scales as $\left(n_{0} a^{3}\right)^{1 / 2}$. At our highest density, that correction is $\epsilon \simeq 0.5$. Twobody collisions are subject to an analogous correction but the corresponding $\epsilon$ is approximately 3 times smaller (the corresponding $n_{0}^{3 / 2}$ dependence of $\Gamma$ is far too small to explain the curvature in the data of Fig. 3). In addition, the fact that the sample probably contains a small thermal component means that collisions between the condensed and the thermal parts must be taken into account $[9,6]$. Assuming 


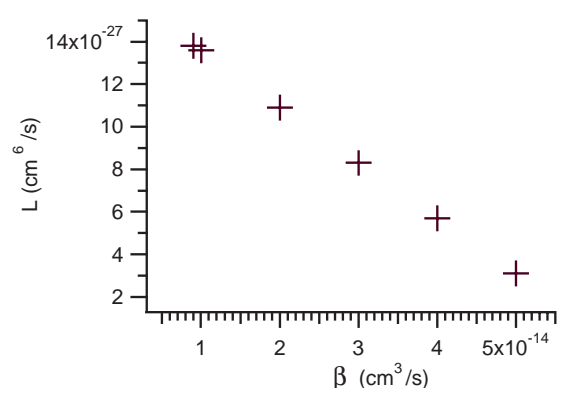

Figure 4: Doublets of fitted values of $\beta$ and L, the 2-body and 3-body ionization rate constants. Each doublet gives a good fit to the data of Fig. 3. The values indicated have been corrected for the effect of quantum depletion. The two fitted quantities $\beta$ and $L$ are clearly correlated, and should not be allowed to vary independently in their uncertainty domains.

a $10 \%$ thermal population $\left(\frac{\mu}{k_{b} T} \simeq 1.1\right)$, we find an additional correction $\epsilon^{\prime}$ to $\kappa_{3}$ of about 0.35 for the densest sample [20].

Taking into account all these corrections, the fitted values of the collision rate constants [21] are: $\beta_{20}=2.9( \pm 2.0) \times 10^{-14} \mathrm{~cm}^{3} \mathrm{sec}^{-1}$ and $L_{20}=8.5( \pm 5.3) \times 10^{-27} \mathrm{~cm}^{6}$ $\sec ^{-1}$, where the subscripts refer to the assumed value of $a$. The error bars are the fit uncertainties on each parameter considered separately, but in fact these error bars are highly correlated since if $\beta$ is increased, $L$ must be decreased and vice-versa (Fig. 4 ). To evaluate the uncertainties in any modelling of a ionization signal, this correlation must be taken into account (see section 5).

\section{Total BEC decay vs ionization decay: a con- sistency test}

To test the consistency of our measurements, we have studied the decay of the number of atoms in a quasi-pure condensate (Fig.5). To acquire these data, we held the BEC in the trap in the presence of the rf-shield for varying times. This study involves multiple realizations of a BEC, which may exhibit large fluctuations in the initial atom number. Thanks to our ion rate monitoring, it has been possible to suppress this noise by using the ion signal to select only data corresponding to the same ion rate $500 \mathrm{~ms}$ after the end of the ramp. This time corresponds to $t=0$ in the figure. We have also plotted the predicted decay curve (solid line) corresponding to ionization only. This curve results from a numerical integration of the atom loss due to ionization processes, calculated from the values $\beta_{20}$ and $L_{20}$ found in section 4 . The fact that the error bars on $\beta$ and $L$ are correlated leads to a small uncertainty on the solid curve, that happens to be of the same order of magnitude than the typical error bars on the data. The observed decay agrees fairly well with the solid curve, and ionization apparently accounts for most of the loss. Note also that if the ion detection efficiency were actually lower than we assume, the predicted decay due to ionization would be 


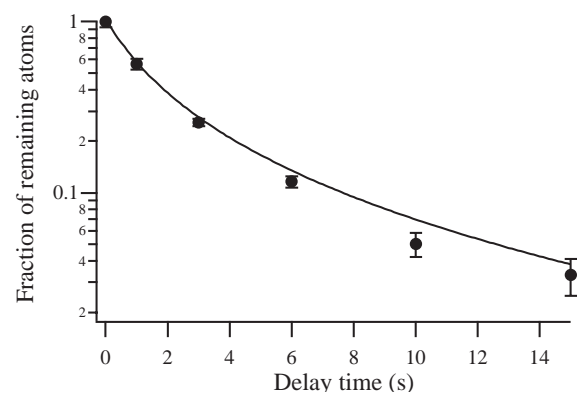

Figure 5: Fraction of remaining atoms measured by TOF as a function of time, for a quasi pure BEC created at $t=0$ and held in the magnetic trap, rf-shield on. The line corresponds to the predicted atom decay according to Eq. 3, with the 2- and 3-body ionization rate constants found in section 4.

faster than the observed decay, an unphysical conclusion. From this, we conclude that our evaluation of the ion detection efficiency is reliable and does not lead to an additional uncertainty in $\beta$ and $L$.

\section{Conclusion and future prospects}

In this presentation, we have shown how collisional ionization of $\mathrm{He}^{*}$ yield an original tool to quantitatively study BEC of this atom. It is indeed possible to continuously monitor the ion current from a trapped sample, without perturbing that sample. By combining such a measurement with time of flight measurements performed at various values of the ion rate, it has been possible to obtain reliable values of the 2-body and 3body ionization rate constants. Now that these values are known, it becomes possible to study the dynamical evolution of a He* condensate by continuously monitoring the ion current. As suggested by the clear take off of the signal visible in Fig.2, the growth of a BEC can be studied with such a method [22].

It might also be possible to study the excitation modes of the condensate. This is particularly interesting since $\mathrm{He}^{*}$ seems well suited to enter the hydrodynamical regime, because of the unusually large scattering length [23]. It is remarkable that although our densest cloud has a mean free path of $\left(8 \pi a^{2} \bar{n}\right)^{-1} \approx 7 \mu \mathrm{m}$, and (using the definition of [8]) has a collisional opacity of $\approx 0.8$, we have no evidence of any avalanche process. This is in contrast to the case of $\mathrm{Rb}$ atoms, where collisional avalanches are a severe hindrance to condensation in the hydrodynamical regime [8].

Because of the large scattering length, quantum depletion is predicted to be large with our densest samples, and has been taken into account to correct the value of the 3-body ionization rate constant. We cannot claim however to have experimentally demonstrated its effect. One way to do so would be to measure the ionization rate vs. the atomic density in thermal clouds, and compare the results to the values found with quasi pure BEC's (section4). Absolute calibrations of ion and atom detection efficiencies should play no role in this comparison, if one could prove that they are the same for both situations. We plan to carry on such a study. 
We thank G. Shlyapnikov for his input about quantum depletion, F. Gerbier for stimulating discussions, A. Villing and F. Moron for their assistance with the electronics. This work is supported by the European Union under grants IST-1999-11055 and HPRN-CT-2000-00125, and by the DGA grant 00.34.025.

\section{References}

[1] A. Robert et al., Science 292, 461 (2001).

[2] F. Pereira Dos Santos et al., Phys. Rev. Lett. 86, 3459 (2001).

[3] G. Shlyapnikov et al., Phys. Rev. Lett. 73, 3247 (1994) ; P. Fedichev et al., Phys. Rev. A 53, 1447 (1996).

[4] H.J. Miesner et al., Science, 270, 1005 (1998); M. Köhl et al., Phys. Rev. Lett. 88, 080402 (2002).

[5] J.L. Roberts et al., Phys. Rev. Lett. 85, 728 (2000).

[6] J. Söding et al., Appl. Phys. B 69, 257 (1999).

[7] E. A. Burt et al., Phys. Rev. Lett. 79, 337 (1997).

[8] J. Schuster et al., Phys. Rev. Lett. 87, 170404 (2001).

[9] Yu. Kagan, B.V. Svistunov, and G.V. Shlyapnikov, JETP Lett. 42, 209 (1985).

[10] A.Browaeys et al., Phys. Rev. A 64, 034703 (2001).

[11] S. Nowak et al., Appl. Phys. B 70, 455 (2000).

[12] A.Browaeys, Thèse de l'université Paris-Sud (2000), available at http://ccsd.cnrs.fr/.

[13] M. O. Mewes et al., Phys. Rev. Lett. 77, 416 (1996).

[14] B. Deconihout et al., Appl. Surf. Sci. 94/95, 422 (1996).

[15] R. S. Gao et al., Rev. Sci. Instrum. 55, 1756 (1984).

[16] V. Venturi et al., Phys. Rev. A 60, 4635 (1999) ; V. Venturi and I. B. Whittingham, Phys. Rev. A 61, 060703(R) (2000).

[17] J. C. Hill et al., Phys. Rev. A 5, 189 (1972).

[18] N. Herschbach et al., Phys. Rev. A, 61, 50702 (2000).

[19] P.O Fedichev, M.W. Reynolds, and G.V. Shlyapnikov, Phys. Rev. Lett. 77, 2921 (1996).

[20] G. Shlyapnikov (private communication).

[21] Collision rate constants are sometimes defined directly for a $\operatorname{BEC}\left(\beta^{\prime}=\beta / 2\right.$ and $\left.L^{\prime}=L / 6\right)$.

[22] More detailed information may be obtained by imaging the $\mathrm{He}^{+}$ions with an electrostatic lens ( P. Schmidt-Bocking, private communication).

[23] See the poster of J. Leonard, E. Jahier et al.. 